\title{
Risk Prediction for Arrhythmias by Heart Rate Deceleration Runs in Patients with Chronic Obstructive Pulmonary Disease
}

This article was published in the following Dove Press journal: International Journal of Chronic Obstructive Pulmonary Disease

\author{
Zhi-Bin Kong' \\ Xing-De Wang (iD ${ }^{2}$ \\ Shu-Rong Shen ${ }^{2}$ \\ Hua Liu' \\ Li Zhou ${ }^{2}$ \\ Bin Chen iD ${ }^{2}$ \\ Zhong Chen ${ }^{2}$ \\ Shao-Jun Yin' \\ 'Department of Respiratory Medicine, \\ Shanghai Sixth People's Hospital East \\ Affiliated to Shanghai University of \\ Medicine and Health Science, Shanghai \\ 201306, People's Republic of China; \\ ${ }^{2}$ Department of Cardiology, Shanghai \\ Sixth People's Hospital East Affiliated to \\ Shanghai University of Medicine and \\ Health Science, Shanghai 201306, \\ People's Republic of China
}

Correspondence: Xing-De Wang Department of Cardiology, Shanghai Sixth People's Hospital East Affiliated to Shanghai University of Medicine and Health Science, No. 222 Huanhu West Road, Lingang New City, Pudong New Area, Shanghai 201306, People's Republic of China

Tel +862I 38297277

Email sd_wangxd@sumhs.edu.cn

Shao-Jun Yin

Department of Respiratory Medicine, Shanghai Sixth People's Hospital East Affiliated to Shanghai University of Medicine and Health Science, No. 222 Huanhu West Road, Lingang New City, Pudong New Area, Shanghai 201306,

People's Republic of China

Tel +862I38297762

Email yinshaojun2010@I63.com
Purpose: Chronic obstructive pulmonary disease (COPD) is associated with increased incidence of arrhythmias, which has been attributed to autonomic dysregulation. Detection of autonomic function may facilitate stratification of COPD patients with respect to their risk of development of arrhythmias.

Patients and Methods: A total of 151 COPD patients and 45 non-COPD patients were included in this study. Heart rate deceleration runs (DRs) were detected by dynamic electrocardiogram (ECG); DRs successively occurring in 2, 4, or 8 cardiac cycles were denoted as $\mathrm{DR}_{2}$, $\mathrm{DR}_{4}$, and $\mathrm{DR}_{8}$, respectively. Indicators of arrhythmias including isolated premature atrial contractions (PAC), supraventricular tachycardia (SVT), isolated premature ventricular contractions (PVC), and ventricular tachycardia (VT) were recorded. Occurrence of SVT or PAC $\geq 70$ /day was considered positive for supraventricular arrhythmias, while positive ventricular arrhythmias category (PVAC) was defined as occurrence of VT or PVC $\geq 10$ /hour.

Results: Compared with non-COPD individuals, COPD patients were associated with increased number of PAC, PVC, higher incidence of PAC >70/d, SVT, PVAC, and decreased DRs (DR2, $\mathrm{DR} 4, \mathrm{DR} 8)(\mathrm{P}<0.05)$. In COPD patients, DRs showed a negative correlation with the incidence of PAC, PVC, SVT, and PVAC $(\mathrm{P}<0.05)$. In receiver operating characteristic curve analysis, all the DRs were found to be significant predictors of PAC $>70 / \mathrm{d}$, SVT, and PVAC. The predictive power of DRs was significantly different from one another with the order ranged as DR4 $>$ DR8 $>$ DR2 for PAC $>70 /$ d, DR8 $>$ DR4 $>$ DR2 for SVT, and DR8 $>$ DR4 $>$ DR2 for PVAC.

Conclusion: Our study provides evidence of significant autonomic dysregulation in COPD patients. DRs may serve as a marker of the risk of arrhythmias in COPD patients.

Keywords: chronic obstructive pulmonary disease, arrhythmias, deceleration capacity runs, autonomic function, risk stratification

\section{Introduction}

Chronic obstructive pulmonary disease (COPD) is one of the most frequently occurring diseases globally. The associated morbidity and mortality burden has shown an increasing trend over the years. Indeed, COPD imposes a heavy economic burden on individual families and societies. ${ }^{1,2}$ The condition is frequently complicated by other diseases, which further exacerbate COPD and increase the associated mortality. ${ }^{3,4}$ Cardiovascular disease is the most common comorbid condition in COPD patients, which usually develops into heart failure and is a major cause of death in these patients. ${ }^{5}$ As reported in previous studies, 4-33\% of COPD patients died of respiratory failure, while $25 \%$ died of heart failure. ${ }^{6}$ Apart from coronary heart disease and 
congestive heart disease, COPD patients commonly develop arrhythmias, which have a significant impact on prognosis. ${ }^{7-11}$ In a study, the incidence of ventricular premature beats $\geq 30$ episodes/hour, paroxysmal ventricular tachycardia, and persistent ventricular tachycardia among COPD patients was $31.9 \%$, $13.0 \%$, and $1.6 \%$, respectively. In addition, the severity of COPD showed an independent correlation with the incidence of paroxysmal ventricular tachycardia. ${ }^{12}$ Detection and prediction of arrhythmias among COPD patients can facilitate better treatment decision-making and prognostic assessment since early detection implies early treatment as well as timely monitoring. However, there are no robust strategies for riskstratification of COPD patients with respect to the development of arrhythmias.

The pathogenetic mechanism of COPD is not completely understood. Quite a few theories had been proposed, including oxidative stress, protease-antiprotease imbalance, and pulmonary inflammation induced by inspiration of noxious particles; these mechanisms are believed to result in the destruction of bronchial structure and development of airflow limitation. Autonomic dysregulation is also believed to play a role in the development of COPD. ${ }^{13,14}$ Autonomic nerves can be classified into two types: sympathetic nerves that promote acceleration capacity of heart rate and the parasympathetic nerves that are closely associated with deceleration capacity of heart rate. Autonomic dysfunction is a common cause of arrhythmias, ${ }^{15}$ which explains the concomitant occurrence of arrhythmia and COPD in most patients.

Previous studies have shown that assessment of deceleration runs (DRs) using the Phase Rectified Signal Averaging (PRSA) technique (a method for quantitative assessment of vagal tone) is an efficient and noninvasive tool to identify patients who are at a high risk of arrhythmiaassociated sudden death. ${ }^{16-18}$ Unlike the quantification of deceleration capacity (DC) based on the average prolonged length of RR intervals, which focuses on the average decrease in intensity of deceleration, DRs quantify cardiac decelerating capability by the frequency of RR elongation, which reflects the regulatory effect of continuous deceleration as a whole. In a study by Arsenos et al, DC was found to be an independent predictor of sudden cardiac death. ${ }^{19}$ In our previous study, patients with acute exacerbation of COPD with concomitant ventricular arrhythmias showed an association with reduced DC, which suggests its value as an indicator of autonomic dysfunction. ${ }^{20} \mathrm{~A}$ recent study also found that DC can help stratify COPD patients based on their risk of arrhythmias. ${ }^{21}$ However, the predictive value of DRs for risk stratification of COPD patients is yet to be clarified. In this study, we sought to evaluate the correlation of DRs with COPD and assess its application for predicting the occurrence of arrhythmias in COPD patients. It is envisaged that our findings may facilitate treatment decision-making and help improve the prognosis of COPD patients in clinical practice.

\section{Patients and Methods}

\section{Study Population}

A total of 151 COPD patients who were hospitalized at the Shanghai Sixth People's Hospital east campus between January 2016 and June 2019 were recruited in this study. Patients were categorized into four subgroups according to COPD stage (I-IV) based on the forced expiratory volume in one second (FEV1) after administration of inhalational bronchodilator. All the included patients qualified the diagnostic criteria of COPD as proposed by the Global initiative for chronic Obstructive Lung Disease (GOLD) in 2013. ${ }^{22}$ Patients with any of the following conditions were excluded: 1) complicated with acute myocardial infarction, chronic heart failure, or thyroid disease; 2) patients with implanted pacemaker; 3) patients with long-term administration of anti-arrhythmic drugs. Another group of 45 non-COPD patients admitted to our hospital during the same period were included as the control group; the age and sex distribution were similar to that of the COPD cohort. All subjects underwent pulmonary function test, ECG, $24 \mathrm{hr}$ dynamic ECG, and echocardiography.

The study was conducted in accordance with the Declaration of Helsinki and approved by the ethics committee of our hospital (ethics committee approval number: 2017-019). All the included subjects were fully informed about the study and provided written informed consent for their participation.

\section{Data Collection}

\section{General Clinical Information}

Data pertaining to variables such as age, sex, height, weight, medical history, comorbid conditions, and medication history were collected from the medical records.

\section{Spirometry}

Pulmonary function of all the included individuals was assessed by spirometry test performed with JAEGER Masterscreen Diffusion. Indicators of pulmonary function including forced vital capacity (FVC) and forced expiratory 
volume in one second (FEV1) after inspiration of bronchodilator were recorded. Patients with FEV1 after inspiration of bronchodilator/FVC $<70 \%$ were diagnosed with COPD. COPD patients were classified into four stages according to criteria issued by GOLD in 2013: 37 patients with FEV1 $\geq 80 \%$ expected value were classified as GOLD1; 40 patients with $50 \%$ expected value $\leq$ FEV1 $<80 \%$ expected value were classified as GOLD2; 44 patients with $30 \%$ expected value $\leq$ FEV1 $<50 \%$ expected value were classified as GOLD3, and 30 patients with FEV1 $<30 \%$ expected value were classified as GOLD4.

\section{Dynamic ECG}

Dynamic ECG was performed for all subjects during the first week of admission. The recorded data was further analyzed by the analytic system of dynamic ECG (DM Software, Stateline, NV, USA). After elimination of interference and artifacts produced by man-machine interaction, the remaining valid records were required to be at least $20 \mathrm{hrs}$; based on the records, the average heart rate (AHR), DRs, and occurrence of arrhythmias were calculated and summarized.

\section{Calculation and Analysis of DRs}

Deceleration runs (DRs) was calculated according to the following steps: 1) interval between two $R$ waves on the ECG record was measured for each subsequent cardiac cycle. 2) A line chart with RR interval as y axis and the number of the corresponding cardiac cycles as $\mathrm{x}$ axis was drawn, which facilitated the detection of DRs (DR2-DR10). DR2 was defined as heart rate deceleration occurring in two sequential cardiac cycles; DR3 was defined as deceleration occurring in three sequential cardiac cycles, and so forth. 3) DRs was calculated as DRs = episodes of DRs in a certain period/total RR number in a certain period.

According to the risk-stratification of arrhythmias proposed by Guzik et al, ${ }^{18}$ patients with DR4 $>0.05 \%$, DR2 $>5.4 \%$, and DR8 $>0.005 \%$ were classified as low-risk group; patients with DR $4>0.05 \%$ but DR $\leq 5.4 \%$ or DR8 $\leq 0.005 \%$ were classified as medium-risk group; patients with DR $4 \leq 0.05 \%$ were classified as high-risk group.

\section{Definition of Arrhythmias}

Arrhythmias were classified into two types: supraventricular arrhythmias and ventricular arrhythmias. Occurrence of one of the following conditions was considered as supraventricular arrhythmias: supraventricular tachycardia (SVT) (including atrial fibrillation, atrial flutter, atrial tachycardias, or atrioventricular junctional tachycardia); isolated premature atrial contraction (PAC) $\geq 70$ beats/day. Occurrence of ventricular tachycardia (VT) or isolated premature ventricular contraction (PVC) $\geq 10$ beats/hour was defined as positive ventricular arrhythmia category (PVAC). VT was defined as occurrence of more than 3 sequential PVCs, with heart rate $>100$ beats/ minute.

\section{Transthoracic Echocardiography}

Transthoracic echocardiography (TTC) was performed using the Philips IE33 Color Doppler Echocardiography (Philips, Amsterdam, The Netherlands). Indicators of cardiac function including left ventricular ejection fraction (LVEF) and pulmonary artery systolic pressure (PASP) were collected.

\section{Statistical Analysis}

Statistical analyses were performed with Graphpad prism 8.0 (GraphPad Software, USA). Normally distributed variables are presented as mean \pm standard deviation, while non-normally distributed variables are presented as median (interquartile range) $[\mathrm{M}(\mathrm{QR})]$. Between-group differences with respect to normally distributed continuous variables were assessed using the unpaired $t$ test, while those with respect to non-normally distributed continuous variables were assessed using the Mann-Whitney $U$-test. Comparison of mean values in multiple groups was carried out by ANOVA analysis for normally distributed continuous variables, and Kruskal-Wallis test for non-normally distributed continuous variables. Two-group comparisons among multiple groups were performed using the post hoc test. Dichotomous or categorical variables are expressed as percentage/ratio, and between-group differences assessed using the Chi-squared or Fisher exact test. Pearson and Spearman correlation tests were used to assess the correlation between normally distributed and non-normally distributed continuous variables, respectively. With DRs as the continuous variables, receiver operating characteristic (ROC) curve analysis was performed for different types of arrhythmias, which were expressed as dichotomous variables. Dependency of specificity on sensitivity was plotted. Area under the curve (AUC) was quantified by integral method. The optimal cut-off value was determined based on the Youden index and used for further analysis. Odds ratios (OR) for arrhythmias were calculated in various COPD subgroups (based on the risk-stratification determined by DRs). For all statistical analyses, $\mathrm{P}$ values $<0.05$ were considered statistically significant. 


\section{Results}

\section{Baseline Characteristics in Different Groups}

As mentioned in the Methods section, we classified the included individuals into five groups: four groups of COPD patients (classified based on GOLD staging criteria) and one group of non-COPD individuals. COPD subgroups of GOLD stage 1-4 accounted for $24.5 \%, 26.5 \%, 29.1 \%$, and $19.9 \%$ of the entire COPD population, respectively. There was no significant difference among the five groups with respect to sex, age, left ventricular ejection function (LVEF), or the prevalence of hypertension, diabetes mellitus, and coronary heart disease $(\mathrm{P}>0.05)$ (Table 1). COPD groups had significantly higher atrial heart rate (AHR) $(\mathrm{P}<0.05)$ and lower FEV1, FVC, FEV1/FVC $(\mathrm{P}<0.0001)$ as compared to non-COPD controls. In addition, the percentage of patients on anticholinergic drugs $(\mathrm{P}=0.0001)$ or steroids $(\mathrm{P}<0.0001)$ in the COPD groups was significantly higher than that in the non-COPD group.

\section{Incidence of Arrhythmias in Different Groups}

The detailed incidence of arrhythmias in different groups is illustrated in Table 2. The P values shown in the table refer to the comparison of the entire COPD group with nonCOPD group. COPD groups had a significantly higher incidence of supraventricular arrhythmias (SVA) and ventricular premature contractions (PVC) as compared to non-COPD control $(\mathrm{P}<0.05)$. Further subgroup analysis demonstrated that only patients with GOLD3 and GOLD4 COPD stage had a significantly higher incidence of $\mathrm{PAC}>70 / \mathrm{d}$ as compared to non-COPD group ( $\mathrm{P}=0.0005$ and 0.0002 , respectively). All COPD subgroups manifested significantly higher incidence of SVT as compared to control $(\mathrm{P}=0.036,0.0234$, 0.0048 , and $<0.0001$, respectively). Incidence of ventricular arrhythmias also tended to be higher in COPD subgroups, though only the difference between GOLD4 and control was statistically significant $(\mathrm{P}=0.006)$.

The correlation of GOLD staging with indicators of arrhythmias, including number of PAC, PVC, incidence of PAC $>70 / d$, SVT, PVAC, was further analyzed; the results showed a positive correlation with all of these indicators $(r=0.3067,0.2255,0.3488,0.3099$, and 0.2644 , respectively ( $\mathrm{P}$ values $<0.0001,0.0015,<0.0001,<0.0001$, and 0.0002 , respectively).

We calculated the ORs for the occurrence of PAC $>70 / \mathrm{d}$, SVT, and PVAC in COPD population as compared with nonCOPD control. COPD patients showed an increased risk of $\mathrm{PAC}>70 / \mathrm{d}[\mathrm{OR}=3.364,95 \%$ CI $(1.554-7.279), \mathrm{P}=0.0018]$, SVT $[\mathrm{OR}=3.875,95 \% \mathrm{CI}(1.827-8.220), \mathrm{P}=0.0003]$ and PVAC [OR=2.186, 95\% CI $(0.8581-5.568), \mathrm{P}=0.1069]$; however, only the results of PAC $>70 / \mathrm{d}$ and SVT were statistically significant.

\section{Deceleration Runs in Different Groups}

As shown in Table 3, indicators of deceleration capacity (DC) including DR2, DR4, DR8 were significantly

Table I Comparison of Baseline Characteristics of Patients in Various Study Groups

\begin{tabular}{|c|c|c|c|c|c|c|}
\hline Parameters & $\begin{array}{l}\text { Non-COPD } \\
(n=45)\end{array}$ & $\begin{array}{l}\text { COPD-GOLDI } \\
(n=37)\end{array}$ & $\begin{array}{l}\text { COPD-GOLD2 } \\
(n=40)\end{array}$ & $\begin{array}{l}\text { COPD-GOLD3 } \\
(n=44)\end{array}$ & $\begin{array}{l}\text { COPD-GOLD4 } \\
(n=30)\end{array}$ & $P$ value \\
\hline Age (years) & $63.15 \pm 7.14$ & $67.30 \pm 9.31$ & $67.85 \pm 8.75$ & $68.84 \pm 7.56$ & $70.53 \pm 6.55$ & 0.0532 \\
\hline Gender (male \%) & $28(62.22)$ & $27(72.97)$ & $29(72.5)$ & $32(72.73)$ & $22(73.33)$ & 0.7569 \\
\hline FEVI/expected FEVI(\%) & $99.22 \pm 5.94$ & $84.30 \pm 6.01$ & $58.21 \pm 6.76$ & $40.01 \pm 5.44$ & $27.36 \pm 4.26$ & $<0.0001$ \\
\hline FVClexpected FVC (\%) & $97.43 \pm 9.46$ & $88.95 \pm 10.2$ & $76.53 \pm 14.47$ & $55.7 I \pm 10.97$ & $48.04 \pm 9.91$ & $<0.0001$ \\
\hline FEVI/FVC (\%) & $89.85 \pm 3.53$ & $65.21 \pm 3.58$ & $59.05 \pm 10.07$ & $56.62 \pm 9.43$ & $45.88 \pm 11.64$ & $<0.0001$ \\
\hline AHR (beats/min) & $71.02 \pm 10.42$ & $72.86 \pm 10.20$ & $73.45 \pm 10.04$ & $74.57 \pm 10.24$ & $78.30 \pm 12.82$ & 0.0274 \\
\hline LVEF (\%) & $61.36 \pm 6.01$ & $60.89 \pm 6.02$ & $61.63 \pm 8.12$ & $59.14 \pm 3.96$ & $58.24 \pm 4.95$ & 0.1918 \\
\hline PASP $(\mathrm{mmHg})$ & $25.97 \pm 10.40$ & $25.92 \pm 5.23$ & $27.24 \pm 6.02$ & $26.85 \pm 3.68$ & $30.47 \pm 7.53$ & 0.2357 \\
\hline Hypertension (\%) & $4(8.89)$ & $4(11.76)$ & $4(10.00)$ & $5(11.36)$ & $4(13.33)$ & 0.9814 \\
\hline Diabetes mellitus (\%) & $2(4.44)$ & $3(8.11)$ & $2(5.00)$ & $3(6.82)$ & $3(10.00)$ & 0.9120 \\
\hline Coronary heart disease (\%) & $2(4.44)$ & $3(8.11)$ & $4(10.00)$ & $4(9.09)$ & $3(10.00)$ & 0.8788 \\
\hline $\begin{array}{l}\text { History of anticholinergic } \\
\text { therapy (\%) }\end{array}$ & $0(0.00)$ & I (2.70) & $6(15.00)$ & $9(20.45)$ & $10(33.33)$ & 0.0001 \\
\hline History of steroid therapy (\%) & $0(0.00)$ & $6(16.21)$ & $30(75.00)$ & $40(90.90)$ & $27(90.00)$ & $<0.0001$ \\
\hline
\end{tabular}

Notes: Data presented as mean \pm standard deviation or frequency (\%). P-values pertain to the difference between patients in the control group versus all patients in the COPD group.

Abbreviations: COPD, chronic obstructive pulmonary disease; GOLD, global initiative for chronic obstructive lung disease; FVC, forced vital capacity; FEVI, forced expiratory volume in the first second; AHR, average heart rate; LVEF, left ventricular ejection fraction; PASP, pulmonary artery systolic pressure. 
Table 2 Comparison of the Incidence of Arrhythmias in Various Study Groups

\begin{tabular}{|c|c|c|c|c|c|c|c|}
\hline & & \multirow{2}{*}{$\begin{array}{l}\text { Non- } \\
\text { COPD } \\
(n=45)\end{array}$} & \multicolumn{4}{|l|}{ COPD } & \multirow[t]{2}{*}{$P$ value } \\
\hline & & & $\begin{array}{l}\text { GOLDI } \\
(n=37)\end{array}$ & $\begin{array}{l}\text { GOLD2 } \\
(n=40)\end{array}$ & $\begin{array}{l}\text { GOLD3 } \\
(n=44)\end{array}$ & $\begin{array}{l}\text { GOLD4 } \\
(n=30)\end{array}$ & \\
\hline \multirow[t]{3}{*}{$\begin{array}{l}\text { Supra-ventricular } \\
\text { arrhythmias }\end{array}$} & $\begin{array}{l}\text { PAC } \\
{[M(Q R)]}\end{array}$ & $\begin{array}{l}138.3 \\
(4.5-60.5)\end{array}$ & $\begin{array}{l}291.9 \\
(4.5-204.0)\end{array}$ & $\begin{array}{l}626.8 \\
(5.0-255.8)\end{array}$ & $\begin{array}{l}881.2 \\
(19.0-569.0)\end{array}$ & $\begin{array}{l}1425 \\
(20.8-1589)\end{array}$ & 0.0025 \\
\hline & PAC> 70/d(n, \%) & $10(22.2)$ & $12(32.4)$ & $16(40.0)$ & $26(59.1)$ & $20(66.7)$ & 0.0018 \\
\hline & SVT (n, \%) & II (24.4) & I 8 (48.6) & $20(50.0)$ & $24(54.5)$ & $22(73.3)$ & $<0.0001$ \\
\hline \multirow[t]{2}{*}{ Ventricular arrhythmias } & $\begin{array}{l}\text { PVC } \\
{[M(Q R)]}\end{array}$ & $\begin{array}{l}81.5 \\
(0.0-4.5)\end{array}$ & $\begin{array}{l}195.6 \\
(0.5-119.5)\end{array}$ & $\begin{array}{l}270.7 \\
(0.25-124.0)\end{array}$ & $\begin{array}{l}819.6 \\
(0.0-240.8)\end{array}$ & $\begin{array}{l}1048.0 \\
(1.0-956.3)\end{array}$ & 0.0015 \\
\hline & PVAC (\%) & $6(13.3)$ & $5(13.5)$ & $9(22.5)$ & II (25.0) & $13(43.3)$ & 0.1069 \\
\hline
\end{tabular}

Notes: Data presented as mean \pm standard deviation, frequency (\%), or median (interquartile range). P-values pertain to the difference between patients in the control group versus all patients in the COPD group.

Abbreviations: COPD, chronic obstructive pulmonary disease; GOLD, global initiative for chronic obstructive lung disease; PAC, premature atrial contraction; PVC, premature ventricular contraction; SVT, supraventricular tachycardia; PVAC, positive ventricular arrhythmia category; M (QR), median (interquartile range).

Table 3 Comparison of DRs in Various Study Groups

\begin{tabular}{|c|c|c|c|c|c|c|}
\hline & \multirow[t]{2}{*}{ Non-COPD $(n=45)$} & \multicolumn{4}{|l|}{ COPD } & \multirow[t]{2}{*}{$P$ value } \\
\hline & & GOLDI $(n=37)$ & GOLD2 $(n=40)$ & GOLD3 $(n=44)$ & GOLD4 $(n=30)$ & \\
\hline DR2 (\%) & $7.69 \pm 1.67$ & $7.58 \pm 1.42$ & $7.4 I \pm I .47$ & $7.30 \pm 1.75$ & $5.69 \pm 1.97$ & 0.0427 \\
\hline DR4 & 0.66 & 0.49 & 0.44 & 0.27 & 0.22 & $<0.0001$ \\
\hline [M (QR),\%] & $(0.39-0.87)$ & $(0.33-0.56)$ & $(0.28-0.59)$ & $(0.12-0.35)$ & $(0.086-0.34)$ & \\
\hline DR8 & 0.25 & 0.017 & 0.013 & 0.056 & 0.0043 & $<0.0001$ \\
\hline [M (QR),\%] & $(0.0075-0.04)$ & $(0.002-0.03)$ & $(0.00-0.02)$ & $(0.00-0.0058)$ & $(0.00-0.0057)$ & \\
\hline \multicolumn{7}{|c|}{ Risk Stratification Based on DRs } \\
\hline Low risk (\%) & $38(84.5)$ & $25(67.6)$ & $18(45.0)$ & $13(29.5)$ & $7(23.3)$ & $<0.0001$ \\
\hline Intermediate risk+ & $7(15.5)$ & $12(32.4)$ & $22(55.0)$ & $31(70.5)$ & $23(76.7)$ & $<0.0001$ \\
\hline High risk (\%) & & & & & & \\
\hline
\end{tabular}

Notes: Data presented as mean \pm standard deviation, frequency (\%), or median (interquartile range). P-values pertain to the difference between patients in the control group versus all patients in the COPD group.

Abbreviations: $\mathrm{DR}_{2}, \mathrm{DR}_{4}$, and $\mathrm{DR}_{8}$, episodes of 2, 4, or 8 consecutive beat-to-beat heart rate decelerations, respectively; $\mathrm{M}$ (QR), median (interquartile range); COPD, chronic obstructive pulmonary disease; GOLD, global initiative for chronic obstructive lung disease.

lower in the COPD population as compared to nonCOPD control $(\mathrm{P}<0.05)$.

Subgroup analysis was also performed to compare the difference between COPD subgroups as well as between COPD subgroups and non-COPD control. Significantly reduced level of DR2 was observed in the GOLD4 subgroup as compared with GOLD1-GOLD3 subgroups as well as the non-COPD group $(\mathrm{P}<0.001)$. A similar phenomenon was observed for DR4; the difference between GOLD4/GOLD3 and non-COPD subgroups ( $\mathrm{P}<0.0001)$ as well as that between GOLD4 and GOLD1/GOLD2 ( $\mathrm{P}<0.0001$ and $\mathrm{P}=0.01$, respectively) were found to be statistically significant. As for DR8, the difference between
GOLD4/GOLD3 and non-COPD subgroups $(\mathrm{P}<0.0001)$ as well as that between GOLD4 and GOLD1/GOLD2/GOLD3 $(\mathrm{P}<0.05)$ were also found to be statistically significant.

The subjects were classified into low-risk group, intermediate-risk group, and high-risk groups according to DRs. As demonstrated in Table 3, COPD patients had a higher risk of being classified in the low-risk group [OR 5.185, 95\% CI (2.071-12.98), $\mathrm{P}<0.001]$. Due to few high-risk cases in the control group, we combined the intermediate-risk and high-risk cases in a single group. The incidence of intermediate-risk plus high-risk cases in the COPD group was 7.58 times higher than that in the control group [OR 7.583, 95\% CI (3.181-18.08), $\mathrm{P}<0.001]$. 
Table 4 Correlation Between Deceleration Capacity and the Incidence of Arrhythmias

\begin{tabular}{|l|l|l|l|l|l|l|l|l|}
\hline \multirow{2}{*}{ Parameters } & \multicolumn{2}{l}{ PAC } & \multicolumn{2}{l}{ PVC } & \multicolumn{2}{l|}{ SVT } & \multicolumn{2}{l|}{ PVAC } \\
\cline { 2 - 9 } & $\mathbf{r}$ & $\mathbf{P}$ & $\mathbf{r}$ & $\mathbf{P}$ & $\mathbf{r}$ & $\mathbf{P}$ & $\mathbf{r}$ \\
\hline DR2 & -0.3064 & 0.0001 & -0.2102 & 0.0096 & -0.2059 & 0.0112 & -0.2944 & 0.0002 \\
DR4 & -0.5103 & $<0.0001$ & -0.3310 & $<0.0001$ & -0.3106 & 0.0001 & -0.2966 & 0.0002 \\
DR8 & -0.1653 & 0.0425 & -0.2389 & 0.0031 & -0.3019 & 0.0002 & -0.2362 & 0.0035 \\
\hline
\end{tabular}

Abbreviations: PAC, premature atrial contraction; PVC, premature ventricular contraction; SVT, supraventricular tachycardia; PVAC, positive ventricular arrhythmia category.

\section{Relationship Between Deceleration Capacity and Incidence of Arrhythmias in COPD Group}

As illustrated above, the COPD group showed lower DC and a higher incidence of arrhythmias as compared to control; we further evaluated the correlation between DC and the incidence of arrhythmias in COPD patients. The levels of DR2, DR4, and DR8 showed a negative correlation with the incidence of PAC, PVC, SVT, and PVAC $(\mathrm{P}<0.05)$ (Table 4).

We performed ROC curve analysis to assess the predictive power of DRs for PAC $>70 / \mathrm{d}$, SVT, and PVAC among COPD patients. Based on the AUC value, the order of predictive power was DR4 $>$ DR8 $>$ DR2 for $\mathrm{PAC}>70 / \mathrm{d}$, DR8 $>$ DR4 $>$ DR2 for SVT, and DR8 $>$ DR4 $>$ DR2 for PVAC (Figure 1). The differences between the predictive power of different DRs for PAC $>70 / \mathrm{d}$, SVT, and PVAC were statistically significant. On integrating the results of SVT and PVAC, the optimal cutoff value for DR2, DR4, and DR8 was $6.35 \%, 0.485 \%$, and $0.0054 \%$, respectively (Table 5).

We performed risk-stratification of COPD patients based on DRs. Patients in the intermediate- and high-risk groups showed a significantly increased risk of PAC $>70 / \mathrm{d}$ $[\mathrm{OR}=5.17, \quad 95 \% \quad \mathrm{CI} \quad(1.226-21.83), \quad \mathrm{P}=0.0303], \quad \mathrm{SVT}$
[OR=4.13,95\% CI (1.109-15.38), $\mathrm{P}=0.0379]$, and PVAC [OR=10.67, 95\% CI (1.582-71.94), $\mathrm{P}=0.0179]$.

\section{Discussion}

COPD patients commonly develop autonomic dysregulation, ${ }^{11,13}$ which contributes to the development of arrhythmias. ${ }^{23}$ Detection of deceleration capacity based on the PRSA technique has been shown to be superior to the conventional techniques like heart rate variability for evaluation of autonomic nervous function. ${ }^{16-18} \mathrm{HRV}$ represents dysregulation of the autonomic control of blood pressure and heart rate. However, HRV reflects the function of sympathetic nervous system and vagal nervous system as a whole, but not separately. Therefore, DC and DRs are superior to other techniques as these quantify and reflect the functional status of sympathetic nerve system and vagal nerve system at the same time. To the best of our knowledge, the present study, for the first time, demonstrates the predictive value of DRs for the occurrence of arrhythmias in COPD patients.

COPD patients with no structural heart disease showed significantly reduced DR2, DR4, and DR8 level and higher heart rate as compared with non-COPD controls. These

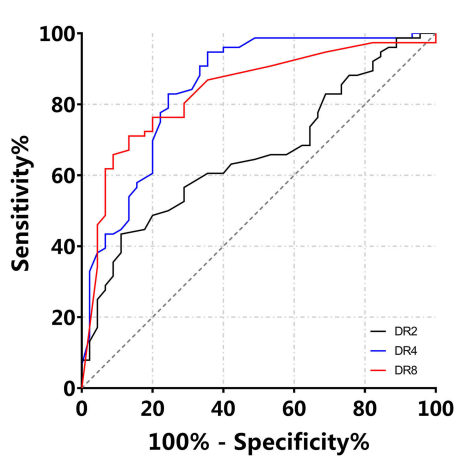

A ROC curve for DRs as predictor for PAC $>70 / d$

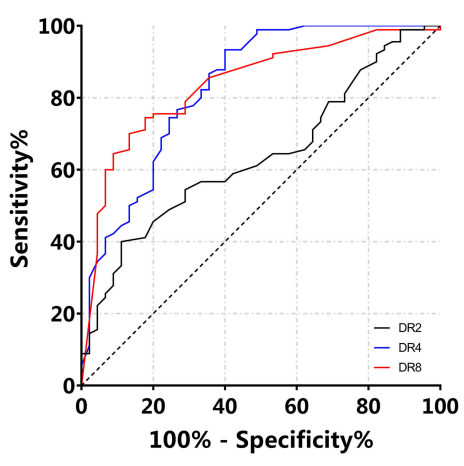

B ROC curve for DRs as predictor for SVT

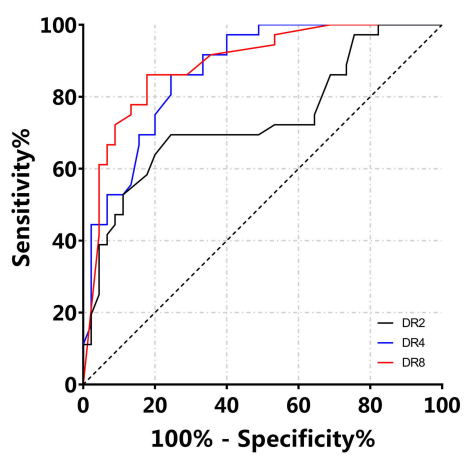

C ROC curve for DRs as predictor for PVAC

Figure I Relationship between DRs and arrhythmias in the COPD group. (A) The order of AUC of ROC curve is DR4>DR8>DR2 for PAC >70/d; (B) the order of AUC of ROC curve is DR8>DR4>DR2 for SVT; (C) the order of AUC of ROC curve is DR8>DR4>DR2 for PVAC.

Abbreviations: COPD, chronic obstructive pulmonary disease; AUC, area under the curve; ROC, receiver operating characteristic. 
Table 5 Comparison of Area Under the ROC Curve for Predicting Arrhythmias Based on DRs

\begin{tabular}{|c|c|c|c|c|c|c|}
\hline & & AUC $(95 \% \mathrm{Cl})$ & Cutoff (\%) & Sensitivity (\%) & Specificity (\%) & $\mathbf{P}$ \\
\hline \multirow[t]{3}{*}{ DR2 } & $\mathrm{PAC}>70 / \mathrm{d}$ & $\begin{array}{l}0.6589 \\
(0.5619-0.7560)\end{array}$ & $<6.25$ & 43.42 & 88.89 & 0.0036 \\
\hline & SVT & $\begin{array}{l}0.6375 \\
(0.5428-0.7323)\end{array}$ & $<6.15$ & 40.00 & 88.89 & 0.0093 \\
\hline & PVAC & $\begin{array}{l}0.7352 \\
(0.6218-0.8486)\end{array}$ & $<6.55$ & 69.44 & 75.56 & 0.0003 \\
\hline \multirow[t]{3}{*}{ DR4 } & PAC $>70 / d$ & $\begin{array}{l}0.8488 \\
(0.7742-0.9234)\end{array}$ & $<0.505$ & 94.74 & 64.44 & $<0.001$ \\
\hline & SVT & $\begin{array}{l}0.8298 \\
(0.7528-0.9067)\end{array}$ & $<0.565$ & 93.33 & 60.00 & $<0.001$ \\
\hline & PVAC & $\begin{array}{l}0.8725 \\
(0.7986-0.9465)\end{array}$ & $<0.405$ & 86.11 & 75.56 & $<0.001$ \\
\hline \multirow[t]{3}{*}{ DR8 } & $\mathrm{PAC}>70 / \mathrm{d}$ & $\begin{array}{l}0.8360 \\
(0.76 \mid 3-0.9107)\end{array}$ & $<0.0049$ & 71.05 & 86.67 & $<0.001$ \\
\hline & SVT & $\begin{array}{l}0.8380 \\
(0.7672-0.9088)\end{array}$ & $<0.0049$ & 70.00 & 86.67 & $<0.001$ \\
\hline & PVAC & $\begin{array}{l}0.8998 \\
(0.8179-0.9617)\end{array}$ & $<0.0059$ & 86.11 & 82.22 & $<0.001$ \\
\hline
\end{tabular}

Abbreviations: ROC, receiver operating characteristic; AUC, area under the curve; PAC, premature atrial contraction; PVC, premature ventricular contraction; SVT, supraventricular tachycardia; PVAC, positive ventricular arrhythmia category; $\mathrm{Cl}$, confidence interval.

findings were indicative of impaired autonomic function in COPD patients, which manifested as reduced vagal activity, depressed deceleration capacity, and increased heart rate. On subgroup analysis, COPD patients with higher GOLD stage tended to have lower levels of DRs, with the lowest level achieved in group GOLD 4; this indicated a correlation between autonomic dysfunction and deterioration of pulmonary function.

Guzik et al proposed DRs-based stratification criteria to classify patients based on the low risk, intermediate risk or high risk of developing arrhythmias. ${ }^{18}$ Based on these criteria, COPD patients in the present study were found to be at a higher risk of developing arrhythmias. Likewise, further subgroup analysis did reveal a higher prevalence of arrhythmias in COPD patients. Similarly, the number of PAC/PVC or incidence of PAC $>70 / \mathrm{d}$, SVT, and PVA showed a positive correlation with GOLD stage; patients with higher GOLD stage experienced higher incidence of arrhythmias. In other words, progression of airway limitation is liable to exacerbate arrhythmias and eventually increase the risk of COPD-associated mortality. All these findings indicate a close correlation of DRs with the incidence of arrhythmias; thus DRs may serve as a useful marker for stratification of COPD patients based on the risk of developing arrhythmias.

It was back in 2006 that DC was first proposed as a predictor of sudden cardiac death in patients with myocardial infarction. ${ }^{17}$ In addition, DC-based risk-stratification criteria were also established. Six years later, use of DRs, as detected by a non-invasive technique (PRSA), was first reported by Guzik et al. They found that DRs were reliable markers for predicting the incidence of sudden death and mortality in patients with myocardial infarction. ${ }^{18}$ The mortality risk predicted by DRs was found to be in perfect agreement with the follow-up results. Both DC and DRs represent quantitative evaluation of the same biological regulatory mechanism of heart rate, but from different perspectives. While DC represents the reduction in the average intensity of each single deceleration in heart rate, DRs reflect the continuous process of deceleration after vagal stimulation. Use of DC and DRs has been widely investigated in many other clinical settings; however, its most common application is for risk-stratification for developing arrhythmias. Arsenos et al validated the application of DC for predicting the risk of arrhythmias in patients with heart failure; they found that $\mathrm{DC} \leq 3.352 \mathrm{~ms}$ was significantly 
associated with increased incidence of arrhythmias. ${ }^{19}$ Buzea et al further evaluated the ability of DC to predict the development of arrhythmias in COPD patients; they also found a significant correlation between $\mathrm{DC}$ and the incidence of arrhythmias. ${ }^{21}$ Based on the results of ROC curve analysis in our study, DRs were found to be a robust predictor of supraventricular and ventricular arrhythmias. Furthermore, different DRs (DR2, DR4, and DR8) demonstrated different sensitivity and specificity for predicting the incidence of different types of arrhythmias. These findings indicate that different DRs may connote different biological significance with respect to cardiac electrophysiological activity. While both DR4 and DR8 were found to efficiently predict supraventricular arrhythmias, DR8 was found to be a better predictor of ventricular arrhythmias. Although we proposed an optimal cutoff level for each DRs for prediction of different arrhythmias, it should be kept in mind that detection of DRs is still a step-up technique that has not been verified in a larger sample size; therefore, the criteria proposed in our study may not be entirely generalizable. Studies conducted in different ethnic groups may also yield different results. A large-scale study on different population is required to establish standard stratification criteria for further clinical application.

Our study confirmed that COPD patients had reduced DRs, which were associated with increased risk of arrhythmia and sudden death. Our results indicated that regulating the autonomic nervous function and improving myocardial electrical stability are important to prevent arrhythmia and sudden death in COPD patients, in addition to improving ventilation, anti-inflammation, and anti-arrhythmia treatment.

Our study demonstrates the feasibility and potential reliability of use of DRs for stratification of COPD patients based on their risk of developing arrhythmia. However, some limitations of our study should be considered while interpreting the results. First, this was a single-center study with a moderate sample size, which may result in selection bias to some extent. In addition, a proportion of COPD patients had a history of treatment with anticholinergic drugs and steroids, which can exacerbate the autonomic dysfunction in patients with stable $\mathrm{COPD}^{24}$ Some of the patients had heart failure with a preserved ejection fraction, which may affect the cutoff level for each DRs. Although there is no evidence regarding the effect of these drugs on PRSA detection, the confounding effect caused by drug administration should be taken into consideration. Besides, the correlation between deceleration capacity and the incidence of arrhythmias was low to moderate in the present study. Many factors can result in arrhythmia including heredity, neuroendocrine diseases, and physiological factors. The diagnosis of arrhythmia requires the combination of DRs, clinical manifestations, and other auxiliary examinations, such as ECG and electrophysiological examination. Last but not the least, the non-normal distribution of some continuous variables as well as the limited representation of certain ethnic groups in our study may have introduced an element of bias. Our findings should be validated in a multi-center study with a larger sample size.

In conclusion, our study demonstrated significant autonomic dysfunction in COPD patients. In addition, COPD patients were found to have an increased risk of arrhythmias, which showed a significant correlation with GOLD stage of COPD. DRs, as a quantitative measure of autonomic dysfunction, may help predict the risk of arrhythmias in COPD patients. Both DR4 and DR8 efficiently predicted the risk of supraventricular arrhythmias, whereas DR8 was found to be a better predictor of ventricular arrhythmias. Our study demonstrates the feasibility of the use of DRs for risk-stratification of COPD patients with respect to the risk of development of arrhythmias.

\section{Acknowledgment}

This study was funded by the clinical innovative project of Shanghai Hospital Development center (SHDC12016238) and the Seed foundation of the Shanghai University of Medicine and Health Science (HMSF-17-22-022). The authors thank Professor Jianping Ye (Shanghai Jiao Tong University Affiliated Sixth People's Hospital) for his guidance on study design and manuscript preparation.

\section{Disclosure}

The authors declare that they have no conflicts of interest in this work.

\section{References}

1. Clini EM, Beghe B, Fabbri LM. Chronic obstructive pulmonary disease is just one component of the complex multimorbidities in patients with COPD. Am J Respir Crit Care Med. 2013;187:668-671. doi:10.1164/rccm.201302-0230ED

2. Vogelmeier CF, Criner GJ, Martinez FJ, et al. Global strategy for the diagnosis, management, and prevention of chronic obstructive lung disease 2017 report: GOLD executive summary. Arch Bronconeumol. 2017;53:128-149. doi:10.1016/j.arbres.2017.02.001

3. Smith MC, Wrobel JP. Epidemiology and clinical impact of major comorbidities in patients with COPD. Int J Chron Obstruct Pulmon Dis. 2014;9:871-888. doi:10.2147/COPD

4. Hansell AL, Walk JA, Soriano JB. What do chronic obstructive pulmonary disease patients die from? A multiple cause coding analysis. Eur Respir J. 2003;22:809-814. doi:10.1183/09031936.03.00031403 
5. Zvezdin B, Milutinov S, Kojicic M, et al. A postmortem analysis of major causes of early death in patients hospitalized with COPD exacerbation. Chest. 2009;136:376-380. doi:10.1378/chest.08-2918

6. Fabbri LM, Luppi F, Beghe B, Rabe KF. Complex chronic comorbidities of COPD. Eur Respir J. 2008;31:204-212. doi:10.1183/ 09031936.00114307

7. Sin DD, Man SF. Chronic obstructive pulmonary disease as a risk factor for cardiovascular morbidity and mortality. Proc Am Thorac Soc. 2005;2:8-11. doi:10.1513/pats.200404-032MS

8. Goudis CA, Konstantinidis AK, Ntalas IV, Korantzopoulos P. Electrocardiographic abnormalities and cardiac arrhythmias in chronic obstructive pulmonary disease. Int $J$ Cardiol. 2015;199:264-273. doi:10.1016/j.ijcard.2015.06.096

9. Schneider C, Bothner U, Jick SS, Meier CR. Chronic obstructive pulmonary disease and the risk of cardiovascular diseases. Eur J Epidemiol. 2010;25:253-260. doi:10.1007/s10654-010-9435-7

10. Conen D, Adam M, Roche F, et al. Premature atrial contractions in the general population: frequency and risk factors. Circulation. 2012;126:2302-2308. doi:10.1161/CIRCULATIONAHA.112.112300

11. Mohammed J, Meeus M, Derom E, Da Silva H, Calders P. Evidence for autonomic function and its influencing factors in subjects with COPD: a systematic review. Respir Care. 2015;60:1841-1851. doi:10.4187/respcare.04174

12. Konecny T, Park JY, Somers KR, et al. Relation of chronic obstructive pulmonary disease to atrial and ventricular arrhythmias. $\mathrm{Am}$ J Cardiol. 2014;114:272-277. doi:10.1016/j.amjcard.2014.04.030

13. van Gestel AJ, Steier J. Autonomic dysfunction in patients with chronic obstructive pulmonary disease (COPD). J Thorac Dis. 2010;2:215-222. doi:10.3978/j.issn.2072-1439.2010.02.04.5

14. Maclay JD, MacNee W. Cardiovascular disease in COPD: mechanisms. Chest. 2013;143:798-807. doi:10.1378/chest.12-0938

15. Andreas S, Haarmann H, Klarner S, Hasenfuss G, Raupach T. Increased sympathetic nerve activity in COPD is associated with morbidity and mortality. Lung. 2014;192:235-241. doi:10.1007/ s00408-013-9544-7
16. Bauer A, Barthel P, Muller A, et al. Risk prediction by heart rate turbulence and deceleration capacity in postinfarction patients with preserved left ventricular function retrospective analysis of 4 independent trials. J Electrocardiol. 2009;42:597-601. doi:10.1016/j. jelectrocard.2009.07.013

17. Bauer A, Kantelhardt JW, Barthel P, et al. Deceleration capacity of heart rate as a predictor of mortality after myocardial infarction: cohort study. Lancet. 2006;367:1674-1681. doi:10.1016/S01406736(06)68735-7

18. Guzik P, Piskorski J, Barthel P, et al. Heart rate deceleration runs for postinfarction risk prediction. J Electrocardiol. 2012;45:70-76. doi:10.1016/j.jelectrocard.2011.08.006

19. Arsenos P, Manis G, Gatzoulis KA, et al. Deceleration capacity of heart rate predicts arrhythmic and total mortality in heart failure patients. Ann Noninvasive Electrocardiol. 2016;21:508-518. doi:10. 1111/anec. 12343

20. Wang X, Jiang Z, Chen B, et al. Cardiac autonomic function in patients with acute exacerbation of chronic obstructive pulmonary disease with and without ventricular tachycardia. BMC Pulm Med. 2016;16:124. doi:10.1186/s12890-016-0287-0

21. Buzea CA, Dan GA, Dan AR, et al. Deceleration and acceleration capacities in risk stratification for arrhythmias in patients with chronic obstructive pulmonary disease. Am J Ther. 2017;24:e44e51. doi:10.1097/MJT.0000000000000450

22. Vestbo J, Hurd SS, Agusti AG, et al. Global strategy for the diagnosis, management, and prevention of chronic obstructive pulmonary disease: GOLD executive summary. Am J Respir Crit Care Med. 2013;187:347-365. doi:10.1164/rccm.201204-0596PP

23. Shen MJ, Zipes DP. Role of the autonomic nervous system in modulating cardiac arrhythmias. Circ Res. 2014;114:1004-1021. doi:10.1161/CIRCRESAHA.113.302549

24. Yuan W, Nie S, Wang H, Xu Q, Jia N. Anticholinergics aggravate the imbalance of the autonomic nervous system in stable chronic obstructive pulmonary disease. BMC Pulm Med. 2019;19:88. doi:10.1186/ s12890-019-0848-0

\section{Publish your work in this journal}

The International Journal of COPD is an international, peer-reviewed journal of therapeutics and pharmacology focusing on concise rapid reporting of clinical studies and reviews in COPD. Special focus is given to the pathophysiological processes underlying the disease, intervention programs, patient focused education, and self management protocols. This journal is indexed on PubMed Central, MedLine and CAS. The manuscript management system is completely online and includes a very quick and fair peer-review system, which is all easy to use. Visit http://www.dovepress.com/testimonials.php to read real quotes from published authors. 